\title{
A novel long non-coding RNA LSAMP-1 is down-regulated in non- small cell lung cancer and predicts a poor prognosis
}

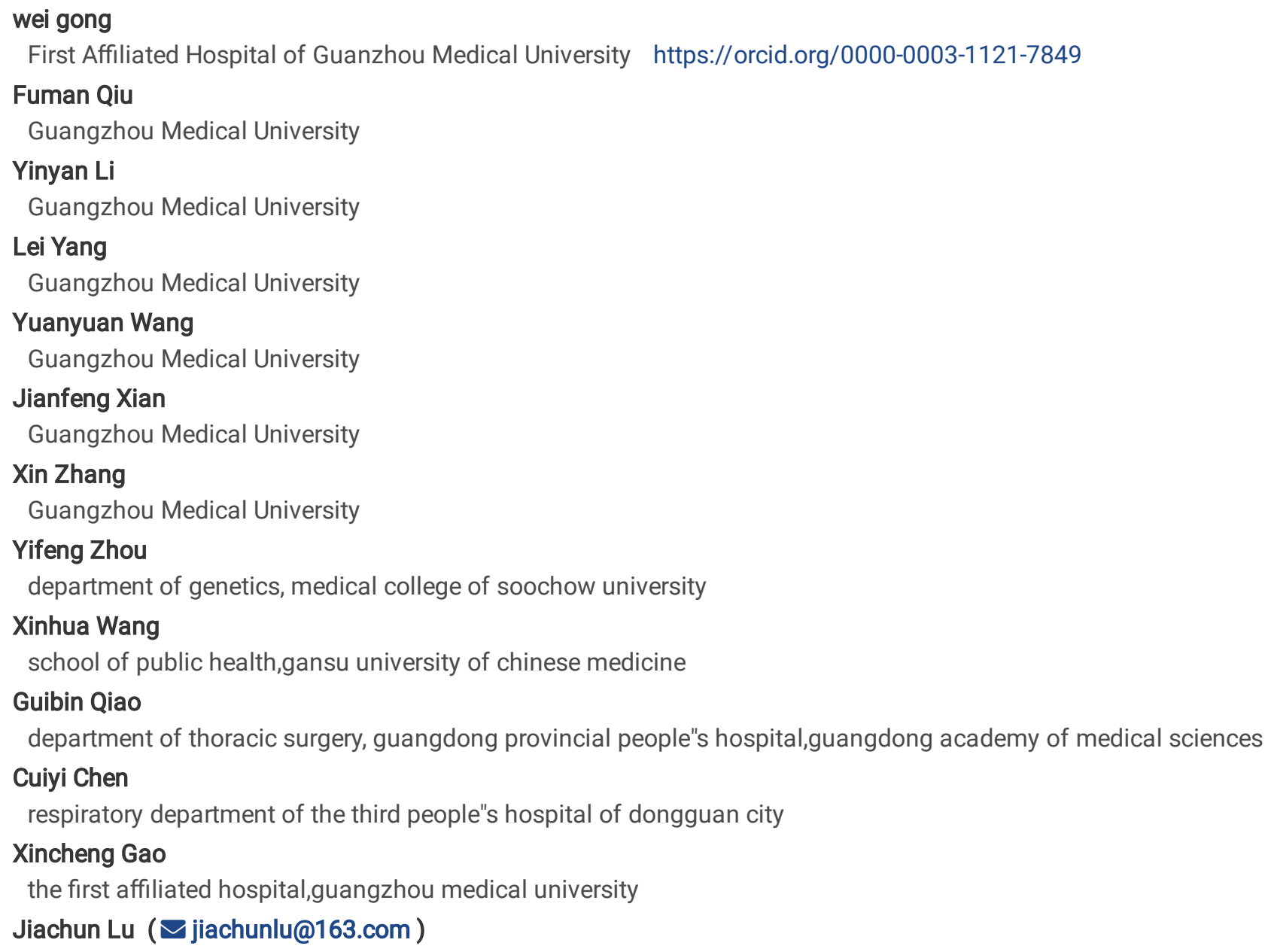

\section{Research article}

Keywords: Lnc-LSAMP-1, LSAMP gene, Non-small cell lung cancer (NSCLC), Biomarker, Prognosis, Clinical pathological features Posted Date: January 17th, 2020

DOI: https://doi.org/10.21203/rs.2.21107/v1

License: @ (1) This work is licensed under a Creative Commons Attribution 4.0 International License. Read Full License 


\section{Abstract}

Background

There is very little known about how long non-coding RNAs (IncRNAs) are associated with membrane proteins in lung cancer. The limbic system-associated membrane protein ( LSAMP) has been reported to play a tumor suppressor role in a variety of cancers.

Methods

We aimed to explore the IncRNA associated with LSAMP and explore its effects on lung cancer.

Results

We found that Lnc-LSAMP-1 was significantly down-regulated in 170 cases of lung tumor tissues when compared to the adjacent non-cancerous tissues $(p<0.001)$. Our results indicated that low expression of Lnc-LSAMP-1 was significantly correlated with stage $(T N M)(p=0.006), N$ status $(p=0.009)$ and poor prognosis $(p=0.004)$. Further investigation showed that overexpression of Lnc-LSAMP1 significantly inhibited lung cancer cell proliferation, viability, invasion and migration ability, arrested cell cycle and facilitated apoptosis. Meanwhile, the expression of Lnc-LSAMP-1 is highly correlated with the expression of nearby tumor suppressor gene LSAMP in our samples $(r=0.7074, p<0.001)$ and TCGA database $(r=0.78, p<0.001)$. It was found that overexpression of Lnc-LSAMP-1 can slow down the degradation rate of LSAMP gene by mRNA protection experiments. By knocking down of LSAMP gene, it was found that overexpressed Lnc-LSAMP-1 cells showed a high proliferation rate. Chemotherapy sensitization experiments showed that overexpression of Lnc-LSAMP-1 enhanced TKI inhibition of lung cancer cell proliferation, which is probably related to affecting the prognosis of patients.

Conclusions

Consequently, the above data suggested that Lnc-LSAMP-1 functions as a tumor suppressor and provides a new potentially therapeutic and prognostic target for non-small cell lung cancer.

\section{Background}

Lung cancer is one of the most common malignant tumors and has become the number one cause of cancer-related deaths in the world[1, 2]. The past 50 years saw a significant increase in the incidence and mortality due to lung cancer in many countries[3]. There are 1.8 million people diagnosed with lung cancer each year, of which in 1.6 million people, it leads to death[4]. Non-small cell lung cancer (NSCLC) is responsible for approximately $85 \%$ of all new lung cancer cases[5]. Despite current technological advancement in clinical and experimental oncology for NSCLC, the overall five-year survival rate for the disease in different regions and countries is between $4-17 \%$, which is still at a low level[6-8]. Molecular biomarkers for potential lung cancer diagnosis and potential gene therapy targets are a recent research focus in the field of lung cancer $[9,10]$. An in depth understanding of the carcinogenesis is crucial for the development of diagnostic markers and new treatment options for anticancer therapy[11].

Recent studies have shown that non-coding RNA (ncRNA) plays a pivotal regulatory role in gene expression[12-15]. LncRNAs (Iong noncoding RNA), important new members of the ncRNA family, are not only modulators of apoptosis and invasion, but also participate in the occurrence and development of cancer[16-18]. For example, IncRNA FEZF-AS1 is associated with advanced clinical stages and family history of lung cancer in patients with NSCLC[19]. LncAGER-1 has an inhibitory effect on lung cancer development by regulating gene AGER[20]and IncRNA IGFBP4-1 reprograms energy metabolism to promote lung cancer progression[21]. MicroRNAs (miRNAs) are also a class of ncRNAs. There are a lot of IncRNA promoting lung cancer by regulating miRNAs[22].

With the help of bioinformatics analysis, we discovered that the low expression of Lnc-LSAMP-1 in lung cancer tissues was associated with poor survival rate of NSCLC patients. We also found that Lnc-LSAMP-1 was down-regulated in 170 cases of lung cancer tissues compared to that in adjacent non-cancerous tissues $(p<0.001)$. Lnc-LSAMP-1 is located on the human chromosome 3q13.32 (chr3: 117391001-117716439) approaching LSAMP gene, which is related to the onset of cancer[23-25]. However, the specific functions of Lnc-LSAMP-1 in lung cancer are not yet clear. Mounting evidence suggested that both self-sufficiency in growth signals and insensitivity to antigrowth signals are among the ten characteristics of tumor[26] and it is known that signal transduction between cells plays an important role in the development of cancer[27]. Membrane proteins are also important mediators of cell 
signaling[28]. Consequently, we suspected that the Lnc-LSAMP-1 suppresses the tumor genesis of lung cancer by regulating LSAMP gene and the following results supported our hypothesis.

\section{Materials And Methods \\ Patients and tissue samples}

The protocol was fully approved by the Institutional Medical Ethics Committee of Guangzhou Medical University, Guangzhou Medical University First Affiliated Hospital, Guangzhou Medical University Affiliated Tumor Hospital, and First Affiliated Hospital of Suzhou University. The purpose of this study was explained and written informed consent forms were obtained from all subjects. A total of 170 NSCLC and adjacent non-tumor tissue specimens were obtained from Guangzhou Medical University First Affiliated Hospital, Guangzhou Medical University Affiliated Tumor Hospital, and First Affiliated Hospital of Suzhou University. 170 patients diagnosed with NSCLC, who had never received any therapy before surgery, were recruited. All clinical data, including age, gender, clinical stage, smoking history, infiltration degree, lymph node metastasis and distant metastasis, of these patients were recorded in a database. In addition, tissue specimens were immediately preserved in RNA later Solution (Thermo Fisher Scientific, US) after removal from the body and were stored at $-80^{\circ} \mathrm{C}$ until use.

\section{Real-time quantitative reverse transcription PCR (qRT-PCR)}

Total RNA from 170 paired NSCLC tissues and 11 cell lines were extracted using TRIzol reagent (Invitrogen, Carlsbad, California, USA). The total RNA was reversely transcribed by using commercial kits according to the manufacturer's instructions (TaKaRa, Japan). RTqPCR reaction (DBI, Germany) was performed in the Applied Biosystems 7900 Fast Real-Time PCR system (Applied Biosystems, CA, USA). $\beta$-actin was used as the endogenous control. We found the Lnc-LSAMP-1 sequence from Lncipedia (http://www.Incipedia.org/). With the help of UCSC genome bioinformatics public database (http://genome.ucsc.edu/cgi-bin/hgGateway), we gained access to other related gene sequences. Primer3Plus online primer design software (http://www.bioinformatics.nl/cgi-

bin/primer3plus/primer3plus.cgi) and NCBI online software were used for primer design. The primers were synthesized by Sangon Biotech Ltd (Shanghai, China). The primer sequences of Lnc-LSAMP-1 were 5》- GTGTTTACACCAGGCACGGG-3》 (forward) and 5》AACCCGGACAGACAAGCCTC-3囚 (reverse) respectively. The primer sequences of $\beta$-actin were 5囚-GGCGGCACCACCATGTACCCT-3囚

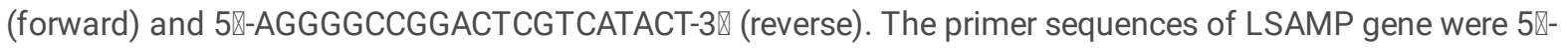

AGAGTTCAGCCGGATCGGAA-3\ (forward) and 5囚-CGTGCCTCGGTTAAAATCCAC-3囚 (reverse). The $2^{-\triangle \triangle C T}$ was used to demonstrate the expression levels of Lnc-LSAMP-1 and LSAMP. All the experiments were conducted in triplicate. The data was analyzed by using the comparative cycle threshold (Ct) method.

\section{Cell culture}

A549 and PC-9 human lung cancer cell lines used in this study were purchased from Cell Bank of Type Culture Collection of the Chinese Academy of Science (Shanghai Institute of Cell Biology, China), in which PC-9 derived from human adenocarcinomas of the lung[29].. Both cell lines were tested and authenticated using a panel of genetic and epigenetic markers before being used in this study. All cells were cultured in RPMI 1640 medium (Gibco, life technologies, California, USA) with 10\% fetal bovine serum (FBS). Cells were placed in a $\mathrm{CO}_{2}$ incubator (SANYO Electric Co., Ltd., Japan) with constant $90 \%$ humidity and $5 \% \mathrm{CO}_{2}$.

\section{Transfection of lentiviral vector}

To generate clones, stably overexpressing Lnc-LSAMP-1, A549 and PC-9 cells were first infected with a lentiviral vector (iGeneBio Co, Ltd, Guangzhou, China) encoding full-length human Lnc-LSAMP-1 sequence and an empty lentiviral vector as the control. Stable clones were screened for 2 weeks using puromycinand the expression level of Lnc-LSAMP-1 was determined by quantitative RT-PCR (qRT-PCR).

\section{Cell proliferation assay}

Cell proliferation assay was performed with Cell Counting Kit-8 (CCK-8, Corning Corporation, USA). Logarithmic phase cells were seeded into 96 -well plates and were cultured for 12, 24,36, and $48 \mathrm{~h}$, respectively. The absorbance of each well was read on a Thermo Scientific ${ }^{\text {TM }}$ VarioskanTM LUX plate reader (Thermo Instruments, USA) (detection wavelength was $450 \mathrm{~nm}$, reference wavelength was $600 \mathrm{~nm}$ ). We also used a dynamic cell viewer for detection abiding by the manufacturer's protocols (IncuCyte ZOOM. Essen BioScience Co., Ltd., USA). 


\section{Flow cytometric analysis of cell cycle and apoptosis}

The flow cytometry analysis was used to identify whether Lnc-LSAMP-1 influenced cell cycle and apoptosis. For cell cycle analysis, stable transfected cells in the logarithmic growth phase were harvested $48 \mathrm{~h}$ after transfection by trypsinization and the propidium iodide staining solution was used for staining. Then the flow cytometric assays (FACScan; BD Biosciences, Shanghai, China) were performed according to the manufacturer's instructions.

For cell apoptosis analysis, Annexin V/7-AAD apoptosis detection kit was used (MultiSciences, HangZhou, China) following the protocol. Cells were washed twice in PBS and re-suspended in $1 \times$ Binding Buffer to achieve a cell concentration of $1.0 \times 10^{6}$ cells $/ \mathrm{ml}$. Subsequently, $10 \mathrm{ul}$ of 7-AAD reagent and $5 \mathrm{ul}$ of Annexin $\mathrm{V}$ reagent were added into cell suspension and stored for $30 \mathrm{~min}$ at room temperature in dark place. Apoptotic cells were examined and quantified using flow cytometry (Becton Dickinson, Lincoln Park, NJ, USA).

\section{Colony-formation assay}

For plate colony formation assay, cells were trypsinized into single cells and seeded into 6-well plates at a density of 200 cells/well. After 10 days of culture, cell clones that had formed from individual cells were directly observed by eye and then the colonies were fixed and stained with Crystal violet, followed by air-drying. The plate colony formation efficiency equals (number of colonies/inoculated cells) $\times 100 \%$. These experiments were performed in triplicate.

\section{Transwell assays}

For the Transwell migration assay, a total of $2 \times 10^{4}$ post-transfection A549 and PC-9 cells were plated in a serum-free medium in the top chamber with a non-coated membrane (24-well insert, pore size $8 \mu \mathrm{m}$; Corning, NY, USA) and a medium supplemented with $10 \%$ serum was in the lower chamber. The invasion experiment was done using the Corning BD BioCoat Matrigel Invasion chamber with a cell diameter of $8 \mu \mathrm{m}$ according to the manufacturer's protocol. After 48 hours, the bottom of the chamber was fixed using formaldehyde for 30 minutes and then stained with $0.1 \%$ crystal violet for 30 minutes. Ten fields were randomly selected under a $100 x$ microscope and the number of cells that migrated to the lower layer was counted. The migration procedure is identical to the invasion experiment procedure without coating with Matrigel matrix. Each assay was performed in triplicate.

\section{Tumorigenicity assay in nude mice}

Animal experiments were approved by the Animal Ethics Committee of Guangzhou Medical University. The number of mice in each group was 5. $200 \mu \mathrm{l}$ of stably transfected lung cancer cells and control cells $\left(1 \times 10^{7}\right.$ cells/ml, A549, PC-9) were injected into the flanks of four-week -old female nude mice (Beijing Huarongkang Biotechnology Co. Ltd). Tumor growth was examined every 3 days for at least one month by measuring the length and width of the tumor mass.

The experimental procedure for tumor metastasis in nude mice is similar to tumor growth model in nude mice. But the injection site of tumor cells is in the tail vein of nude mice. After continuous observation for 6 weeks, the nude mice were killed, and the number of lung cancer metastasis was observed. HE slices were made following relevant protocols and strict operating procedures after soaking and fixing with $4 \%$ paraformaldehyde. The program was approved by the Animal Ethics Committee of Guangzhou Medical University.

\section{Hematoxylin-eosin (HE) staining}

Tumor tissues were fixed in $10 \%$ formalin, embedded in paraffin and then cut into 3 - $\mu \mathrm{m}$-thick sections that were baked at $45^{\circ} \mathrm{C}$ for $5 \mathrm{~h}$. Sections were then stained with HE (artificial hematoxylin and eosin) according to the following steps: 30 min of xylene dewaxing, treated with ethanol at different concentrations (100\%, $90 \%, 70 \%)$, hydrated in distilled water, stained with hematoxylin ( $15 \mathrm{~min}$ ), differentiated in hydrochloric acid ethanol and ammonia water, dehydrated with ethanol at $70 \%$ and $90 \%$ concentrations (10 min), stained with eosin ethanol (3 min), dehydrated with ethanol and cleared with xylene, and tumor tissue sections were then observed under a microscope.

\section{Actinomycin D inhibits RNA synthesis experiments}

Over-expressed A549 and PC-9 cells and control were seeded at $10 \mathrm{~mm} \star 24$-well plates at $5 \times 10^{4}$ per well. After 24 hours, the cells were treated with actinomycin $D$ (the concentration of actinomycin D was $2 \mathrm{mg} / \mathrm{L}$ ). After 30 minutes, 1 hour, 2 hours, 3 hours and 4 hours, the expression levels of LSAMP gene was detected by qRT-PCR. 


\section{Rescue experiment}

The Rescue experiment was performed to validate that Lnc-LSAMP-1 regulated lung cancer cell biology functions by targeting LSAMP. LSAMP inhibitor and blank inhibitor were transferred intoA549 and PC-9 cells. The efficiency of siRNA was measured by qRTPCR. It was observed in Incucyte zoom (Essen BioScience Co., Ltd., USA) that the proliferation of A549 and PC-9 cells led to the inhibition of LSAMP.

\section{Drug sensitivity test of Inc-LSAMP-1 on cisplatin and TKI (Tyrosine kinase inhibitors)}

The IC50 of A549 and PC-9 cells was first screened using cisplatin concentration gradient $(10 \mathrm{ug} \mathrm{/} \mathrm{ml,} 5 \mathrm{ug} / \mathrm{ml}, 2.5 \mathrm{ug} / \mathrm{ml}, 1.25 \mathrm{ug} /$ ml, 0.625 ug / ml, 0.3125 ug / ml). A549: IC505ug / ml; PC-9: IC502.5ug / ml. Nilotinib (TKI) was used as mean plasma drug concentrations such as Nilotinib $=3.6 \mathrm{umol} / \mathrm{L}$. High expression of Inc-LSAMP-1 and control cells were treated with cisplatin and Nilotinib. The high expression group and the control group were inoculated into a 96-well plate, and the number of cells per well was $5.0 \times 10^{3}$. Nilotinib was added after adhering to the wall and growing to about $10 \%$, cisplatin was added up to $30 \%-40 \%$, and the 96 well plate was placed in a dynamic cell observer for detection (IncuCyte ZOOM. Essen BioScience Co. Ltd. USA). The rate of cell proliferation was tested.

\section{Statistical analysis}

All statistical data were analyzed using the SPSS 16.0 software (SPSS, Chicago, USA). The paired-t test was used to assess the differences between lung cancer tissues and adjacent lung normal tissues in regards to gene expression and frequency of methylation level. The $\chi 2$ test was applied to analyze gene expression levels in Table 1 and table $S 1$. The association between LncLSAMP-1 expression and the clinical pathological parameters of NSCLC was assessed by the one-way analysis of variance (ANOVA). Kaplan-Meier and Cox regression analyses were used to assess the association between LnC-LSAMP-1 and overall survival rate as well as the prognosis of NSCLC. Additionally, $\mathrm{P}<0.05$ was considered statistically significant $\left({ }^{\star}, \mathrm{p}<0.05 ;{ }^{*}, \mathrm{p}<0.01 ;{ }^{* \star *}, \mathrm{p}<0.001\right)$. 
Table 1

Associations between the expression of Lnc-LSAMP-1 and clinical feathers as well as demographics among lung cancer patients

\begin{tabular}{|c|c|c|c|c|c|c|c|c|c|}
\hline \multirow[t]{2}{*}{ Characteristic } & \multicolumn{3}{|c|}{ Southern samples $\mathbf{N}(\%)$} & \multicolumn{3}{|c|}{ Eastern samples $\mathbf{N}(\%)$} & \multicolumn{3}{|l|}{ Total N (\%) } \\
\hline & $\begin{array}{l}\text { Low } \\
\text { expression }\end{array}$ & $\begin{array}{l}\text { High } \\
\text { expression }\end{array}$ & $\begin{array}{l}P \\
\text { value }\end{array}$ & $\begin{array}{l}\text { Low } \\
\text { expression }\end{array}$ & $\begin{array}{l}\text { High } \\
\text { expression }\end{array}$ & $\begin{array}{l}P \\
\text { value }\end{array}$ & $\begin{array}{l}\text { Low } \\
\text { expression }\end{array}$ & $\begin{array}{l}\text { High } \\
\text { expression }\end{array}$ & $\begin{array}{l}\mathrm{P} \\
\text { value }\end{array}$ \\
\hline \multicolumn{10}{|l|}{ Age } \\
\hline$<60$ & $40(69.0)$ & 18(31.0) & 0.155 & 19(63.3) & 11(36.7) & 0.311 & $59(67.0)$ & $29(33.0)$ & 0.508 \\
\hline$>=60$ & $32(56.1)$ & $25(43.9)$ & & 19(76.0) & $6(24.0)$ & & $51(62.2)$ & $31(37.8)$ & \\
\hline \multicolumn{10}{|l|}{ Gender } \\
\hline Female & 21(67.7) & $10(32.3)$ & 0.489 & $10(62.5)$ & $6(37.5)$ & 0.722 & $31(66.0)$ & $16(34.0)$ & 0.833 \\
\hline Male & $51(60.7)$ & 33(39.3) & & 28(71.8) & 11(28.2) & & $79(64.2)$ & $44(35.8)$ & \\
\hline \multicolumn{10}{|c|}{ Family tumor history } \\
\hline No & $62(60.8)$ & $40(39.2)$ & 0.407 & $36(70.6)$ & 15(29.4) & 0.767 & $98(64.1)$ & $55(35.9)$ & 0.593 \\
\hline Yes & $10(76.9)$ & $3(23.1)$ & & $2(50.0)$ & $2(50.0)$ & & 12(70.6) & $5(29.4)$ & \\
\hline \multicolumn{10}{|l|}{ Smoking } \\
\hline No & $25(64.1)$ & 14(35.9) & 0.813 & 11(73.3) & $4(26.7)$ & 0.929 & $36(66.7)$ & 18(33.3) & 0.715 \\
\hline Yes & $47(61.8)$ & $29(38.2)$ & & $27(67.5)$ & 13(32.5) & & $74(63.8)$ & $42(36.2)$ & \\
\hline \multicolumn{10}{|l|}{ Stage(TNM) } \\
\hline $\mid+\|$ & $35(74.5)$ & $12(25.5)$ & 0.029 & 16(84.2) & $3(15.8)$ & 0.078 & $51(77.3)$ & 15(22.7) & 0.006 \\
\hline III + IV & $37(54.4)$ & $31(45.6)$ & & $22(61.1)$ & 14(38.9) & & $59(56.7)$ & $45(43.3)$ & \\
\hline \multicolumn{10}{|l|}{ T status } \\
\hline $1+2$ & $47(66.2)$ & $24(33.8)$ & 0.312 & $13(54.2)$ & $11(45.8)$ & 0.035 & $60(63.2)$ & $35(36.8)$ & 0.635 \\
\hline $3+4$ & $25(56.8)$ & 19(43.2) & & $25(80.6)$ & 6(19.4) & & $50(66.7)$ & 25(33.3) & \\
\hline \multicolumn{10}{|l|}{$\mathrm{N}$ status } \\
\hline 0 & $34(75.6)$ & $11(24.4)$ & 0.021 & $22(75.9)$ & $7(24.1)$ & 0.251 & $56(75.7)$ & 18(24.3) & 0.009 \\
\hline $1+2+3$ & $38(54.3)$ & $32(45.7)$ & & $16(61.5)$ & $10(38.5)$ & & $54(56.3)$ & $42(43.8)$ & \\
\hline \multicolumn{10}{|l|}{ M status } \\
\hline 0 & $54(65.1)$ & 29(34.9) & 0.382 & $23(62.2)$ & 14(37.8) & 0.111 & $77(64.2)$ & $43(35.8)$ & 0.820 \\
\hline 1 & 18(56.3) & 14(43.7) & & 15(83.3) & $3(16.7)$ & & $33(66.0)$ & 17(34.0) & \\
\hline \multicolumn{10}{|l|}{$\begin{array}{l}\text { Histological } \\
\text { classification }\end{array}$} \\
\hline Adenocarcinoma & $39(65.0)$ & $21(35.0)$ & 0.632 & $15(68.2)$ & $7(31.8)$ & 1.000 & $54(65.9)$ & $28(34.1)$ & 0.752 \\
\hline $\begin{array}{l}\text { Squamous } \\
\text { carcinoma }\end{array}$ & $16(55.2)$ & $13(44.8)$ & & $13(68.4)$ & 6(31.6) & & $29(60.4)$ & 19(39.6) & \\
\hline Other types ${ }^{a}$ & $17(65.4)$ & $9(34.6)$ & & $10(71.4)$ & $4(28.6)$ & & $27(67.5)$ & 13(32.5) & \\
\hline
\end{tabular}

Results 


\section{Lnc-LSAMP-1 and LSAMP gene expression in lung cancer}

The demographics and clinical features of studied patients were listed in Table S1. The expression of Lnc-LSAMP-1 at 9 lung cancer cell lines and 4 human immortalized lung normal cell lines $(p=0.0007)$ has been shown in Fig. 1a. We found that Lnc-LSAMP-1 was down-regulated in tumor tissues compared to that in non-tumor tissues in 170 lung cancer cases $(p<0.001$, as shown in Fig. 1b) and LSAMP gene was also down-regulated in 86 cases of lung tumor tissues when compared to that of the adjacent non-cancerous tissues ( $p=0.0314$, as shown in Fig. 1c). Meanwhile, the expression of Lnc-LSAMP-1 was positively associated with LSAMP gene expression in 143 cases of NSCLC tissues ( $r=0.7074, p<0.001$, Fig. 1d). Both A549 and PC-9 expressed a relevant portion of LncLSAMP-1 in nucleus (Fig. 1e). As shown in Fig. 1f, Kaplan-Meier analysis indicated that down-regulation of Lnc-LSAMP-1 expression in tissues was associated with poor survival rate of NSCLC patients $(p=0.004)$, which is also consistent with TCGA database (Fig. S2d). The A549 and PC-9 cells that overexpressed Lnc-LSAMP-1 showed significantly higher levels of LSAMP and Lnc-LSAMP-1 expression compared with that of control groups (Fig. $1 \mathrm{~g}$ and $\mathrm{h}$ ).

\section{Lnc-LSAMP-1 expression is correlated with Stage (TNM) and N status}

We analyzed the relationship between Lnc-LSAMP-1 expression level and clinical features. The expression status of Lnc-LSAMP-1 was classified as "High" or "Low" based on the expression of LNC-LSAMP-1 in lung cancer tissues and in adjacent normal lung tissues. We found that low expression of Lnc-LSAMP-1 was significantly correlated with stage $(T N M)(p=0.006)$ and N status $(p=$ 0.009), which is in accordance with TCGA database (Fig. S2f and g). However, Lnc-LSAMP-1 down-expression was not associated with age, gender, family tumor history, smoking, stage, T status and histological classification (all p >0.05), as shown in Table 1 .

\section{The correlation of Lnc-LSAMP-1 and LSAMP gene was investigated in the GEPIA database}

TCGA database (From GEPIA) analysis revealed that the expression levels of Lnc-LSAMP-1 and LSAMP gene were significantly downregulated in NSCLC compared with that of adjacent normal tissues (all $P<0.05$, Fig. S2a and $b$ ). To further search the target gene for Lnc-LSAMP-1, we analyzed the correlation between Lnc-LSAMP-1 and target gene from GEPIA dataset. We found that the LSAMP gene is the most relevant gene $(R=0.78 P<0.001$, Fig. S2c and S1a). We further analyzed the relationship between Lnc-LSAMP-1 expression and clinical analysis in TCGA and found that Lnc-LSAMP-1 expression was associated with T status $(p=0.0349), N$ status $(p=0.0012)$, and stage (TNM) ( $=0.0049)$ (Fig. S2e, S2f and g). We also found that Lnc-LSAMP-1 is located in the downstream of the LSAMP gene and has partial sequence overlap regions (Fig. S1b). The above results indicate that Lnc-LSAMP-1 is highly correlated with the LSAMP gene.

\section{Down-regulation of Lnc-LSAMP-1 predicts a poor prognosis in NSCLC}

To evaluate the potential prognostic value of Lnc-LSAMP-1, we analyzed RNA-seq data from GEPIA. From a cohort of 472 NSCLC patients, Lnc-LSAMP-1 was highly expressed in the lung cancer tissues of 238 patients and lung cancer tissues of the other 234 patients showed low Lnc-LSAMP-1 expression. We assessed the overall survival rate of patients who were Lnc-LSAMP-1-high or LncLSAMP-1-low using the Kaplan-Meier method. As shown in Fig. S2d, the 20-year survival rate was lower in Lnc-LSAMP-1-low patients comparing to Lnc-LSAMP-1-high patients, indicating that a high level of Lnc-LSAMP-1 was correlated with a long survival time (Log rank $p=0.007, \mathrm{HR}($ high $)=0.66 \mathrm{p}(\mathrm{HR})=0.0076)$, supporting the argument for its utility as a biomarker for NSCLC progression and its potential function for early detection of NSCLC.

\section{Lnc-LSAMP-1 suppresses cell proliferation}

To determine the effect of LnC-LSAMP-1 on the viability and proliferation of lung cancer cell in vitro, CCK-8 and colony formation assay showed that the overexpressed Lnc-LSAMP-1 suppressed the viability of A549 and PC-9 in a time dependent manner $(P<0.05$; Fig. 2b). The same results were observed in the dynamic cell viewer (Fig. 2a) and plate colony formation assay (Fig. 2c).

\section{Lnc-LSAMP-1 affects migration and invasion of A549 and PC-9 cells}

To detect the effect of Lnc-LSAMP-1 on cell migration, the Transwell migration assay was carried out in A549 and PC-9 cell lines. As shown in Fig. 2d, the overexpressed Lnc-LSAMP-1 resulted in attenuated migration of lung cancer cells. The Transwell invasion assay 
results also showed that the cell invasive ability was suppressed inA549 and PC-9 cell lines when cells were transfected with LncLSAMP-1 compared with the results from the control groups (Fig. 2f).

\section{Lnc-LSAMP-1 affects cell cycle and induces apoptosis}

As shown by flow cytometry analysis in Fig. 2e, overexpressed Lnc-LSAMP-1 caused significantly increased apoptosis in A549 and PC-9 cells compared with that of the control groups. Accordingly, flow cytometric analysis showed a decrease in the percentage of cells in the S phase and a marked accumulation in the percentage of cells in the G0/G1 phase in A549 and PC-9 cells (Fig. 2g), compared with that of the control groups.

\section{Lnc-LSAMP-1 inhibits tumor growth in vivo}

As shown in Fig. 3a, b and c, tumor growth in nude mice injected with overexpressed Lnc-LSAMP-1 A549 and PC-9 cells was slower than that in control groups and the tumors were smaller. Accordingly, the number of suspected tumors in the lung of nude mice is less than that of control groups, when mice were injected with overexpressed Lnc-LSAMP-1 A549 and PC-9 cells (Fig. 3d and e). Figure $3 f$ showed a subcutaneous tumor-forming $\mathrm{HE}$ section of nude mice, which is consistent with lung cancer tissue morphology. Figure $3 \mathrm{~g}$ showed a HE slice of suspicious lung nodules in nude mice injected with tail vein injection, which can be observed as lung cancer cell morphology from the enlarged part of the figure (red boxes in Fig. 3g).

\section{The knock down of LSAMP gene promotes cell proliferation rate in vitro}

The efficiency of si (LSAMPgene) was measured by qRT-PCR and the results showed that si- 1 could achieve 70 percent of inhibition (Fig. 4a and b), so we selected si-1 for the following experiments. The results showed that A549 and PC-9 cells were proliferating faster after knocking down of LSAMP gene (Fig. 4c and d). Overexpressed A549 and PC-9 cells and control groups were treated with actinomycin $D$ (the concentration of actinomycin $D$ was $2 \mathrm{mg} / \mathrm{L}$ ). The results suggested that the degradation rate of LSAMP gene in A549 and PC-9 cells of over-expressed Lnc-LSAMP-1 was lower than that of the control groups (Fig. 4e and f), which means that LncLSAMP-1 may have the function of protecting LSAMP gene.

\section{Lnc-LSAMP-1 enhances the susceptibility of TKI}

We compared the sensitizing effect of Lnc-LSAMP-1 on TKI and cisplatin by inhibition rate. Proliferation inhibition rate = (experimental group (dosing) - experimental group) / (control group (dosing) - control group) $\times 100 \%$. In the Nilotinib group, the overexpressed Lnc-LSAMP-1 A549 cell inhibition rate in test group was 1.01 times that of the control group. It's 0.8 times in the PC-9 cells (Fig. 5a, b, c and d). However, the effect was not obvious in the carboplatin group. The over-expressed Lnc-LSAMP-1 A549 cell inhibition rate was 0.10 times that of the control group, and in PC-9 there was no effect (Fig. $5 e, f, g$ and $h$ ).

\section{Discussion}

Mounting evidence indicates that IncRNAs are involved in the development of cancer even if some of their roles in lung cancer were poorly understood[30]. The Lnc-LSAMP-1 is closely related to the development of lung cancer from TCGA database. Firstly, the different level of expression of Lnc-LSAMP-1 in lung cancer tissues and adjacent normal tissues was up to 50 (0.04:2.04. from GEPIA database) times. Secondly, the expression level of Lnc-LSAMP-1 was closely related to the development and prognosis of lung cancer. Encouraged by these results, we highly suspected that Lnc-LSAMP-1 is involved in the development of lung cancer. In our experiments we also discovered that Lnc-LSAMP-1 is markedly down-regulated in lung tumor tissues and cell lines. The expression levels of Lnc-LSAMP-1 in patients with NSCLC were associated with stage (TNM) and N status. Additionally, the low expression of Lnc-LSAMP-1 in lung cancer tissues was associated with poor survival rate of NSCLC patients. Thus, we constructed overexpressed Lnc-LSAMP-1 vector in lung cancer cells (A549, PC-9) using lentivirus. We found that overexpression of Lnc-LSAMP-1 inhibited proliferation, viability, invasion and migration ability, arrested cell cycle and facilitated apoptosis. Therefore, these results indicated that Lnc-LSAMP-1 might be a potential tumor suppressor in lung cancer cells. Moreover, survival analysis suggested that downregulation of Lnc-LSAMP-1 expression in tissues was associated with poor survival rate of NSCLC patients, so we conducted chemotherapy sensitization experiments. The results showed that overexpression of Lnc-LSAMP-1 enhanced TKI inhibition of lung cancer cell proliferation, which is probably related with the prognosis of the lung cancer patients. 
There is still an abundant amount of uncharted IncRNAs remaining to be elucidated in terms of their effects on cancer risk[31]. The chromosome location can provide a meritorious hint to the function of IncRNAs as a lot of IncRNAs have the potential to regulate neighbor-coding genes[32]. When we examined the Lnc-LSAMP-1 gene information, we found that there is a sense of mRNA, which is "LSAMP". The LSAMP gene is located on chr3:115528941-117716095 in front of Lnc-LSAMP-1 and some of their sequences overlap. Pearson correlation analysis showed that the expression of Lnc-LSAMP-1 in lung cancer tissues is highly related to the expression of the gene LSAMP. The correlation is the highest in the TCGA database (figure S1a).

LSAMP (limbic system-associated membrane protein) is a self-binding, antibody-like cell surface adhesion protein[33]. The findings of SanzRL showed that overexpression of the LSAMP gene and NTM suppresses outgrowth from DRG neurons. The LSAMP gene locus is activated through recurrent deletions and has been reported in osteosarcoma[34, 35], acute myeloid leukemia[36], renal carcinoma[37] and ovarian carcinoma[38]. Single nucleotide polymorphism within the first intron of LSAMP gene has recently been shown to be a predictor of prostate cancer-specific mortality[39]. Furthermore, alterations of ZBTB20, GAP43 and GSK3B adjacent to LSAMP genes are less clear but they are suspected to have suppressed cancerous functions[40, 41]. Tale Barøy, et al. also reported that re-expression of LSAMP inhibits the growth of osteosarcoma cells and confirmed that LSAMP has tumor suppressor function[42].

Previous studies showed that LSAMP found in the cortical and subcortical areas of the limbic system play a pivotal role in the regulation of emotional behaviors[43]. LSAMP is one of the four IgLONs that constitute the immunoglobulin superfamily. The IgLONs as cell adhesion molecules, are positively involved in modification of cell-cell recognition[44]. Chen et al. found that LSAMP has been recognized as a translocation breakpoint-spanning gene in familiar clear cell renal cell carcinoma. In addition, he pointed out that the expression of LSAMP protein in cell lines with LSAMP promoter methylation inhibited cell proliferation[37]. Based on this information, LSAMP may function as a tumor suppressor gene in cancer development.

However, the molecular mechanisms behind how Lnc-LSAMP-1 suppresses cell proliferation, cell migration, cell invasion and tumor metastasis remain unclear. To examine the relationship of LSAMP and Lnc-LSAMP-1, we found that LSAMP is the most relevant target gene for LnC-LSAMP-1 in TCGA database, which is consistent with our data. The correlation analysis indicated that the expression of LSAMP is highly related to Lnc-LSAMP-1 in lung cancer tissues. Hence, we suspected that Lnc-LSAMP-1 suppresses proliferation, viability, invasion and migration ability, arrests cell cycle and facilitates apoptosis by regulating LSAMP gene.

\section{Conclusions}

In conclusion, our study demonstrated that LnC-LSAMP-1 is one of the down-regulated LnCRNAs in NSCLC, and showed that LncLSAMP-1 was involved in the prognosis of NSCLC for the first time. Low Lnc-LSAMP-1 expression was associated with poor survival rate of NSCLC patients. Furthermore, the co-expression of Lnc-LSAMP-1 and LSAMP genes in lung cancer cells may affect the lymph node metastasis of lung cancer through cell adhesion. We believe that our results may provide a potentially therapeutic and prognostic target for NSCLC.

\section{Declarations}

\section{Ethics approval and consent to participate}

The study was approved by the Ethics Committee of Guangzhou Medical University and Soochow University.

\section{Consent for publication}

We have obtained consents to publish this paper from all the participants of this study.

\section{Availability of data and materials}

The datasets are available from TCGA (http://cancergenome.nih.gov/).

\section{Competing interests}

The authors have declared no conflicts of interest. 


\section{Funding}

This study was supported by the National Key R\&D Projects (2016YFC0903700), the National Natural Science Foundation of China 81473040, 81673267 (J. Lu), 81273149, 81402753, 81672303 (L. Yang), 81602289, 81872127 (F. Qiu),

Guangdong High School Young Innovative Talents Project ( 2015KQNCX136),

Guangzhou Science Research Program General Project(201707010123),

Guangzhou Municipal Scientific Research Project(1201630073) and Guangzhou Science and Technology Program Pearl River Nova projects Grant 201710010049 (L. Yang). Guangdong education bureau Characteristic innovation project Grants 2015 KTSCX116 (L.Yang) and Guangdong Provincial Major Projects Grants 2014KZDXM046, Yangcheng Scholar Grants 1201541589 (J. Lu).

\section{Authors' contributions}

Jiachun Lu designed the study. Wei Gong performed the most experiments. Yinyan Li analyzed the qRT-PCR results. Fuman Qiu, Mingzhu Lin Jianfeng Xian and Xin Zhang collected the tissue samples. Yuanyuan Wang wrote the paper. Lei Yang, Xiaoxiao Lu, Soham Datta and Yifeng Zhou revised the paper writing.

\section{Acknowledgements}

The authors are grateful for the invaluable support and useful suggestions with other members of the molecular epidemiology lab.

\section{Abbreviations}

IncRNA: long non-coding RNA; LSAMP: limbic system-associated membrane protein; RNA-seq: RNA sequencing; ncRNA: non-coding RNA; TCGA: The Cancer Genome Atlas.

\section{References}

1. Siegel RL, Miller KD, Jemal A. Cancer Statistics, 2017. CA Cancer J Clin. 2017;67:7-30.

2. Shen M, Zhao X, Zhao L, Shi L, An S, Huang G, Liu J. Met is involved in TIGAR-regulated metastasis of non-small-cell lung cancer. Mol Cancer. 2018;17:88.

3. Chen W, Zheng R, Baade PD, Zhang S, Zeng H, Bray F, Jemal A, Yu XQ, He J. Cancer statistics in China, 2015. CA Cancer J Clin. 2016;66:115-132.

4. Youlden DR, Cramb SM, Baade PD. The International Epidemiology of Lung Cancer: geographical distribution and secular trends. J Thorac Oncol. 2008;3:819-831.

5. Detillon D, Driessen EJM, Aarts MJ, Janssen-Heijnen MLG, van Eijck CHJ, Veen EJ. Changes in treatment patterns and survival in elderly patients with stage I non-small-cell lung cancer with the introduction of stereotactic body radiotherapy and video-assisted thoracic surgery. Eur J Cancer. 2018;101:30-37.

6. Zahir ST, Mirtalebi M. Survival of patients with lung cancer, Yazd, Iran. Asian Pac J Cancer Prev. 2012;13:4387-4391.

7. Xiao F, Liu D, Guo Y, Shi B, Song Z, Tian Y, Zhang Z, Liang C. Survival rate and prognostic factors of surgically resected clinically synchronous multiple primary non-small cell lung cancer and further differentiation from intrapulmonary metastasis. $J$ Thorac Dis. 2017;9:990-1001.

8. Matsuda A, Katanoda K. Five-year relative survival rate of lung cancer in the USA, Europe and Japan. Jpn J Clin Oncol. 2013;43:1287-1288.

9. Kohno T, Nakaoku T, Tsuta K, Tsuchihara K, Matsumoto S, Yoh K, Goto K. Beyond ALK-RET, ROS1 and other oncogene fusions in lung cancer. Transl Lung Cancer Res. 2015;4:156-164.

10. Guzman L, Depix MS, Salinas AM, Roldan R, Aguayo F, Silva A, Vinet R. Analysis of aberrant methylation on promoter sequences of tumor suppressor genes and total DNA in sputum samples: a promising tool for early detection of COPD and lung cancer in smokers. Diagn Pathol. 2012;7:87.

11. Wang M, Yuang-Chi Chang A. Molecular mechanism of action and potential biomarkers of growth inhibition of synergistic combination of afatinib and dasatinib against gefitinib-resistant non-small cell lung cancer cells. Oncotarget. 2018;9:1653316546 . 
12. Keller C, Kulasegaran-Shylini R, Shimada Y, Hotz HR, Buhler M. Noncoding RNAs prevent spreading of a repressive histone mark. Nat Struct Mol Biol. 2013;20:994-1000.

13. Filipowicz W, Bhattacharyya SN, Sonenberg N. Mechanisms of post-transcriptional regulation by microRNAs: are the answers in sight? Nat Rev Genet. 2008;9:102-114.

14. Ponting CP, Oliver PL, Reik W. Evolution and functions of long noncoding RNAs. Cell. 2009;136:629-641.

15. Pennisi E. Long noncoding RNAs may alter chromosome's 3D structure. Science. 2013;340:910.

16. Qi P, Du X. The long non-coding RNAs, a new cancer diagnostic and therapeutic gold mine. Mod Pathol. 2013;26:155-165.

17. Lv J, Liu H, Huang Z, Su J, He H, Xiu Y, Zhang Y, Wu Q. Long non-coding RNA identification over mouse brain development by integrative modeling of chromatin and genomic features. Nucleic Acids Res. 2013;41:10044-10061.

18. Gibb EA, Brown CJ, Lam WL. The functional role of long non-coding RNA in human carcinomas. Mol Cancer. 2011;10:38.

19. Gong W, Cao Y, Wang Y, Yang L, Su W, Qiu F, Datta S, Rao B, Xian J, Lin M et al. Upregulation of LncRNA FEZF-AS1 is associated with advanced clinical stages and family history of cancer in patients with NSCLC. Pathol Res Pract. 2018;214:857-861.

20. Pan Z, Liu L, Nie W, Miggin S, Qiu F, Cao Y, Chen J, Yang B, Zhou Y, Lu J et al. Long non-coding RNA AGER-1 functionally upregulates the innate immunity gene AGER and approximates its anti-tumor effect in lung cancer. Mol Carcinog. 2017.

21. Yang B, Zhang L, Cao Y, Chen S, Cao J, Wu D, Chen J, Xiong H, Pan Z, Qiu F et al. Overexpression of IncRNA IGFBP4-1 reprograms energy metabolism to promote lung cancer progression. Molecular cancer. 2017;16:154.

22. Muhammad N, Bhattacharya S, Steele R, Ray RB. Anti-miR-203 suppresses ER-positive breast cancer growth and stemness by targeting SOCS3. Oncotarget. 2016;7:58595-58605.

23. Kjeldsen E. Duplication of subtelomeric regions in an adult with acute monocytic leukemia with an acquired jumping translocation involving 3q13.31-qter. Data Brief. 2017;13:675-682.

24. Herve B, Fauvert D, Dard R, Roume J, Cognard S, Goidin D, Lozach F, Molina-Gomes D, Vialard F. The emerging microduplication 3q13.31: Expanding the genotype-phenotype correlations of the reciprocal microdeletion 3q13.31 syndrome. Eur J Med Genet. 2016;59:463-469.

25. Novak AJ, Asmann YW, Maurer MJ, Wang C, Slager SL, Hodge LS, Manske M, Price-Troska T, Yang ZZ, Zimmermann MT et al. Whole-exome analysis reveals novel somatic genomic alterations associated with outcome in immunochemotherapy-treated diffuse large B-cell lymphoma. Blood Cancer J. 2015;5:e346.

26. Hanahan D, Weinberg RA. Hallmarks of cancer: the next generation. Cell. 2011;144:646-674.

27. Kolch W, Halasz M, Granovskaya M, Kholodenko BN. The dynamic control of signal transduction networks in cancer cells. Nat Rev Cancer. 2015;15:515-527.

28. Manford AG, Stefan CJ, Yuan HL, Macgurn JA, Emr SD. ER-to-plasma membrane tethering proteins regulate cell signaling and ER morphology. Dev Cell. 2012;23:1129-1140.

29. Lee YC, Saijo N, Sasaki Y, Takahashi H, Sakurai M, Ishihara J, Hoshi A, Chen KM, Hamburger AW. Clonogenic patterns of human pulmonary adenocarcinoma cell lines (PC-9, PC-13 and PC-14) and how they influence the results of test for chemosensitivity to cisplatin in the human tumor clonogenic assay. Jpn J Clin Oncol. 1985;15:637-644.

30. Spizzo R, Almeida MI, Colombatti A, Calin GA. Long non-coding RNAs and cancer: a new frontier of translational research? Oncogene. 2012;31:4577-4587.

31. Malek E, Jagannathan S, Driscoll JJ. Correlation of long non-coding RNA expression with metastasis, drug resistance and clinical outcome in cancer. Oncotarget. 2014;5:8027-8038.

32. Kyriakou D, Stavrou E, Demosthenous P, Angelidou G, San Luis BJ, Boone C, Promponas VJ, Kirmizis A. Functional characterisation of long intergenic non-coding RNAs through genetic interaction profiling in Saccharomyces cerevisiae. BMC Biol. 2016;14:106.

33. Kresse SH, Ohnstad HO, Paulsen EB, Bjerkehagen B, Szuhai K, Serra M, Schaefer KL, Myklebost O, Meza-Zepeda LA. LSAMP, a novel candidate tumor suppressor gene in human osteosarcomas, identified by array comparative genomic hybridization. Genes Chromosomes Cancer. 2009;48:679-693.

34. Lee JT. Epigenetic regulation by long noncoding RNAs. Science. 2012;338:1435-1439.

35. Sanchez-Elsner T, Gou D, Kremmer E, Sauer F. Noncoding RNAs of trithorax response elements recruit Drosophila Ash1 to Ultrabithorax. Science. 2006;311:1118-1123. 
36. Kuhn MW, Radtke I, Bullinger L, Goorha S, Cheng J, Edelmann J, Gohlke J, Su X, Paschka P, Pounds S et al. High-resolution genomic profiling of adult and pediatric core-binding factor acute myeloid leukemia reveals new recurrent genomic alterations. Blood. 2012;119:e67-75.

37. Chen J, Lui WO, Vos MD, Clark GJ, Takahashi M, Schoumans J, Khoo SK, Petillo D, Lavery T, Sugimura J et al. The t $(1 ; 3)$ breakpoint-spanning genes LSAMP and NORE1 are involved in clear cell renal cell carcinomas. Cancer cell. 2003;4:405-413.

38. Ntougkos E, Rush R, Scott D, Frankenberg T, Gabra H, Smyth JF, Sellar GC. The IgLON family in epithelial ovarian cancer: expression profiles and clinicopathologic correlates. Clin Cancer Res. 2005;11:5764-5768.

39. Huang SP, Lin VC, Lee YC, Yu CC, Huang CY, Chang TY, Lee HZ, Juang SH, Lu TL, Bao BY. Genetic variants in nuclear factor-kappa B binding sites are associated with clinical outcomes in prostate cancer patients. Eur J Cancer. 2013;49:3729-3737.

40. Chen Z, Liu C, Patel AJ, Liao CP, Wang Y, Le LQ. Cells of origin in the embryonic nerve roots for NF1-associated plexiform neurofibroma. Cancer Cell. 2014;26:695-706.

41. Shi Y, Hu Z, Wu C, Dai J, Li H, Dong J, Wang M, Miao X, Zhou Y, Lu F et al. A genome-wide association study identifies new susceptibility loci for non-cardia gastric cancer at 3q13.31 and 5p13.1. Nat Genet. 2011;43:1215-1218.

42. Baroy T, Kresse SH, Skarn M, Stabell M, Castro R, Lauvrak S, Llombart-Bosch A, Myklebost O, Meza-Zepeda LA. Reexpression of LSAMP inhibits tumor growth in a preclinical osteosarcoma model. Mol Cancer. 2014;13:93.

43. Pimenta AF, Zhukareva V, Barbe MF, Reinoso BS, Grimley C, Henzel W, Fischer I, Levitt P. The limbic system-associated membrane protein is an Ig superfamily member that mediates selective neuronal growth and axon targeting. Neuron. 1995;15:287-297.

44. Eagleson KL, Pimenta AF, Burns MM, Fairfull LD, Cornuet PK, Zhang L, Levitt P. Distinct domains of the limbic system-associated membrane protein (LAMP) mediate discrete effects on neurite outgrowth. Mol Cell Neurosci. 2003;24:725-740.

\section{Supporting Information}

Fig. S1(a)Predictive analysis of Inc-LSAMP-1 target genes in the GEPIA data. (b) The positions of LSAMP gene and Inc-LSAMP-1 overlap partially in the UCSC data.

Fig. S2. (a) (b) Lnc-LSAMP-1 was down regulated in LUAD and LUSC similar to LSAMP gene by GEPIA data. (c) The correlation between $L S A M P$ gene and $L n C-L S A M P-1$ in lung cancer tissues by GEPIA data (d) Kaplan-Meier analysis of patients in low expression of Inc-LSAMP-1 group and control group. (e) (f) (g) Low expression of Lnc-LSAMP-1 was significantly correlated with T status, N status and Stage(TMN) by TCGA data. Significance was defined as $p<0.05(*, p<0.05 ; * \star, p<0.01 ; * \star \star, p<0.001)$.

\section{Figures}



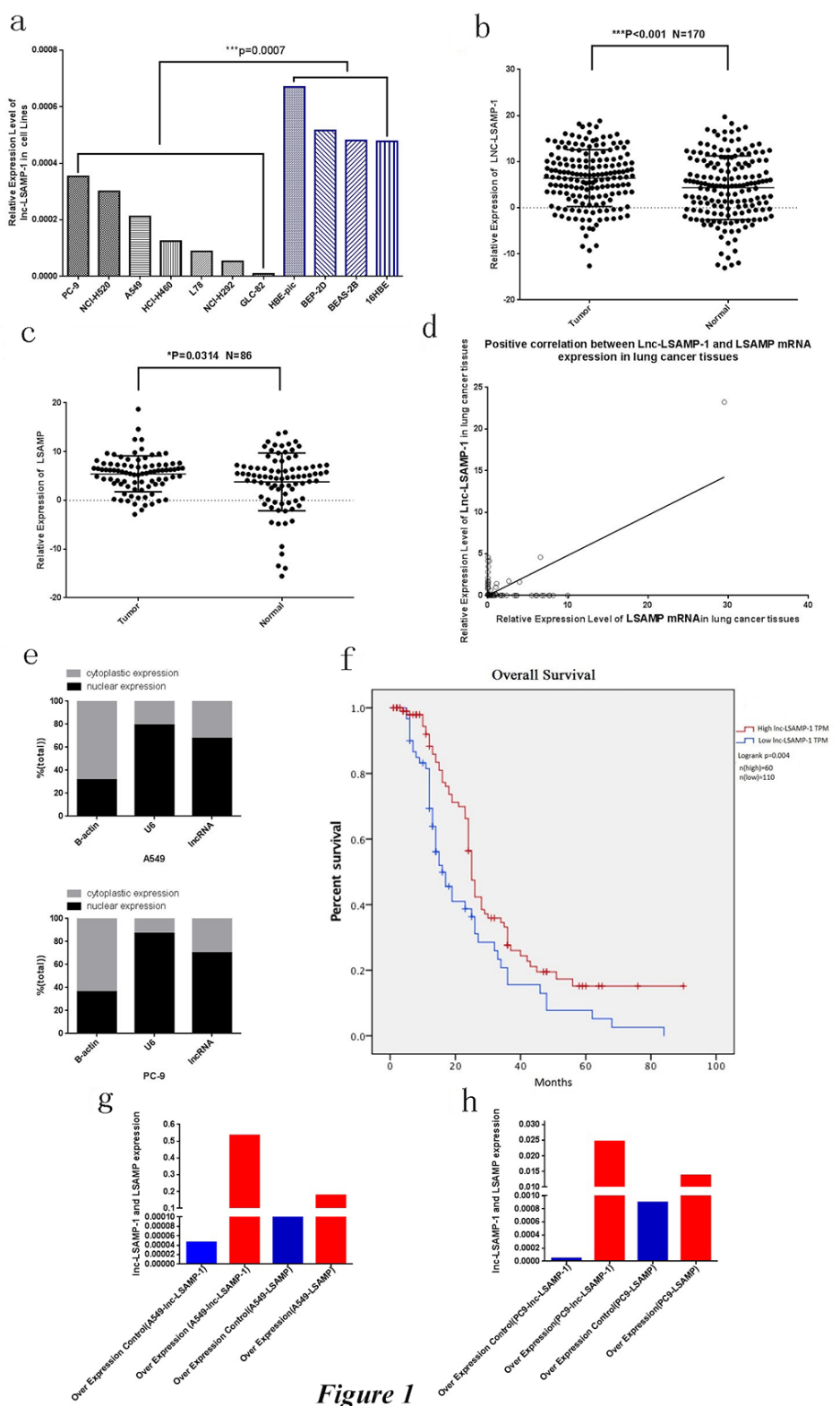

\section{Figure 1}

(a) The expression of Lnc-LSAMP-1 in lung cancer and normal cell lines (b) Lnc-LSAMP-1 expression levels in 170 pair-matched tumor tissues and adjacent normal tissues measured by qRT-PCR. (c) LSAMP gene expression levels in 86 pair-matched tumor tissues and adjacent normal tissues measured by qRT-PCR. (d)The correlation between Lnc-LSAMP-1 and LSAMP gin 143 pairs of lung cancer tissues. (e) Subcellular localization of Lnc-LSAMP-1 expression. (f) Kaplan-Meier analysis of patients in low expression of Inc-LSAMP-1 group and control group. (g) (h) High expression of Lnc-LSAMP-1 and LSAMP gene in A549 and PC-9 cells that transfected with overexpressed Lnc-LSAMP-1. Significance was defined as $p<0.05\left({ }^{\star} p<0.05 ;{ }^{* *} p<0.01 ; * \star * p<0.001\right)$. 

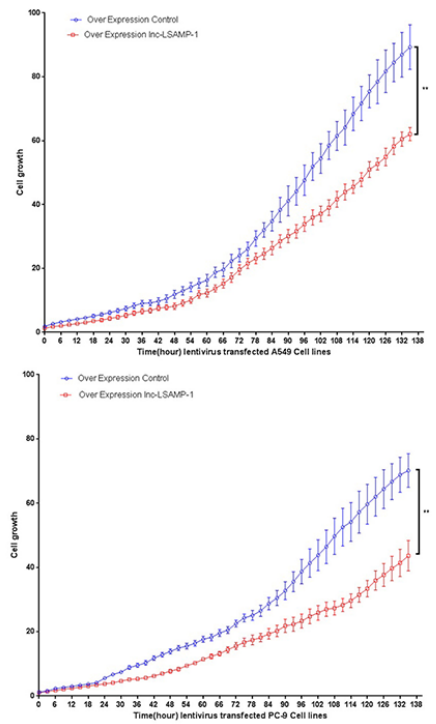

d

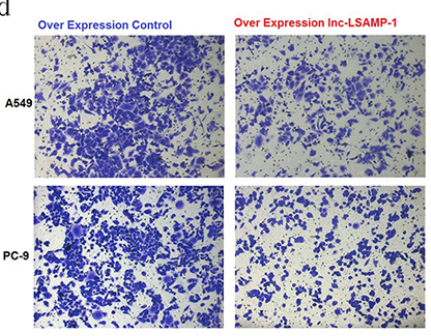

f

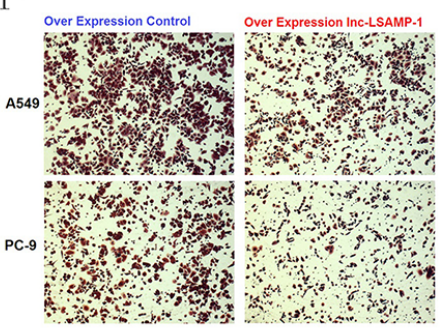

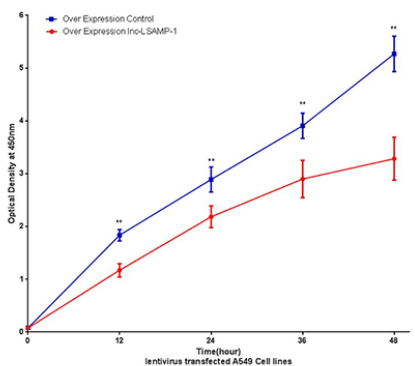

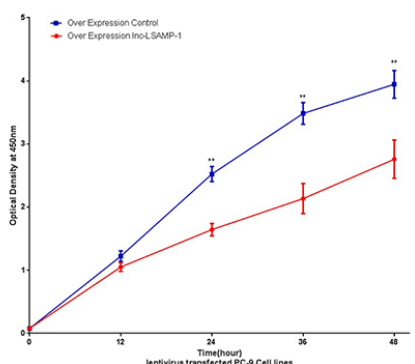

PC-9
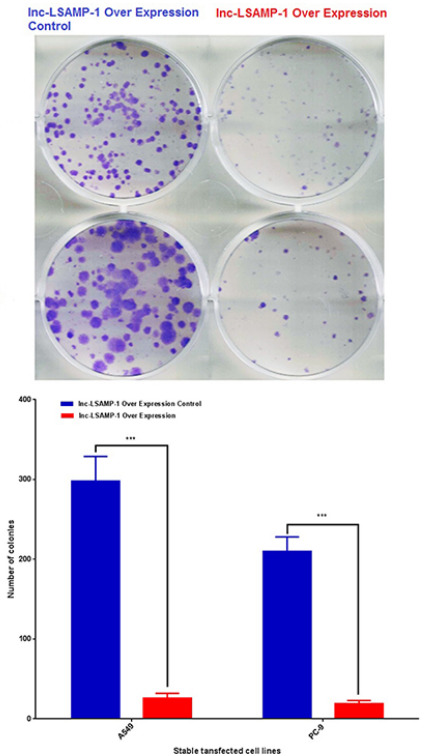

e
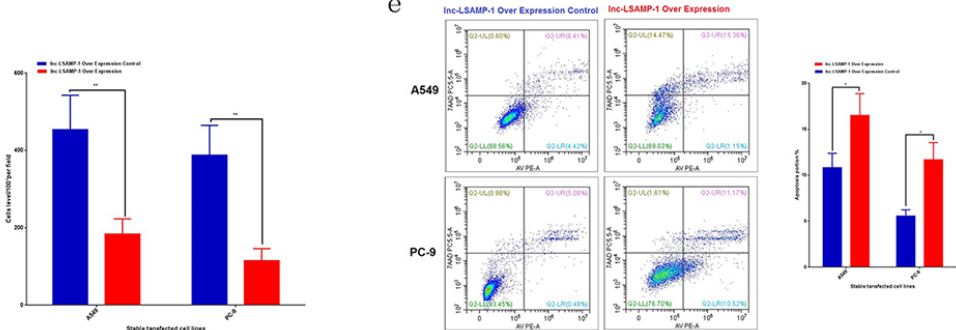

g

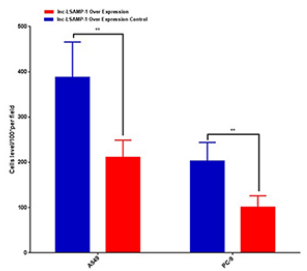

Figure 2

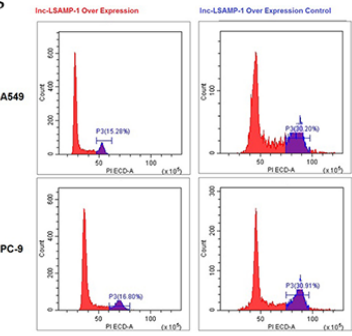

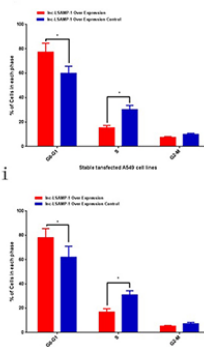

Figure 2

Overexpression of endogenous Lnc-LSAMP-1 inhibits lung cancer cell proliferation in vitro. A549 and PC-9 cells were transfected with overexpressed Lnc-LSAMP-1 vector (pLNC-LSAMP-1) and empty vector as control. (a) Cell proliferation assay in Incucyte zoom (Essen BioScience Co., Ltd., USA). (b) CCK8 assay was performed to determine the cell proliferation. (c) clone formation was performed to determine the cell proliferation. Migration(d) and invasion(f) capacities determined by Transwell assays. (e) The flow cytometry was conducted to determine the cell cycle of A549 and PC-9 cells. (g) The flow cytometry was conducted to determine the cell apoptosis of A549 and PC-9 cells. The results from three independent experiments, showed as mean \pm s.d. Significance was defined as $p<0.05(*, p<0.05 ; * \star, p<0.01 ; * \star *, p<0.001)$. 

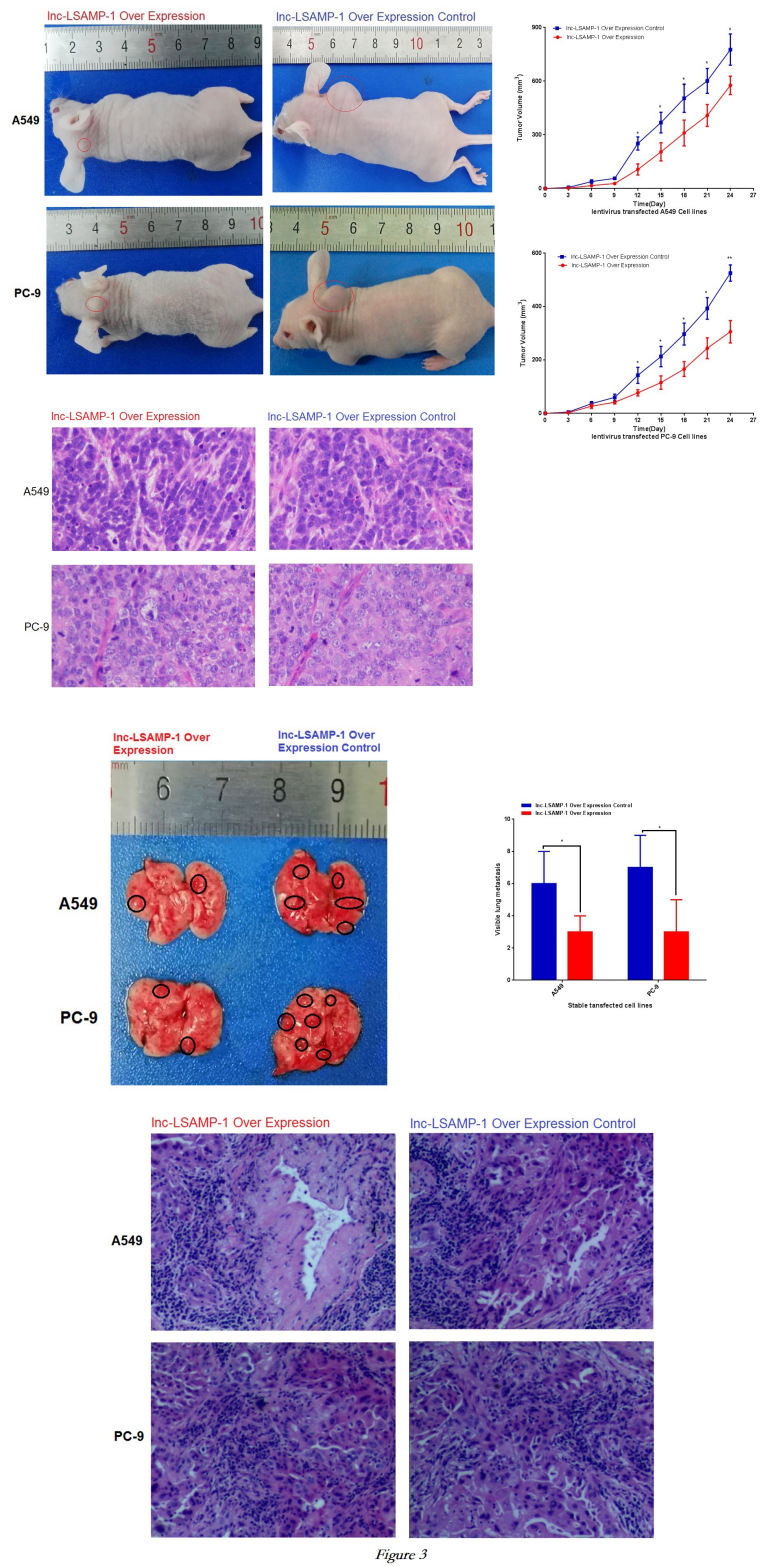

\section{Figure 3}

Overexpression of LnC-LSAMP-1 inhibited proliferation and metastasis of A549 and PC-9 cells in vivo. (a) Nude mice inoculated subcutaneously with A549 and PC-9 cells. (b) (c)Tumor growth rate and tumor volume are compared in Lnc-LSAMP-1 overexpression group and control group. (d) Lung images observed after treatment of nude mice in different groups of the two cell lines and the black circles in the figure refer to suspected metastases.(e) The number of suspected cancerous lesions in the naked eyes of nude mice after sacrificed in nude mice. (f) A subcutaneous tumor-forming HE section of nude mice. (g) A HE slice of suspicious lung nodules in nude mice injected with tail vein injection. Red box shows potential cancer areas. Significance was defined as $p<0.05\left({ }^{*}, p<0.05 ; * \star\right.$, $p<0.01 ; * \star \star, p<0.001)$. 
a

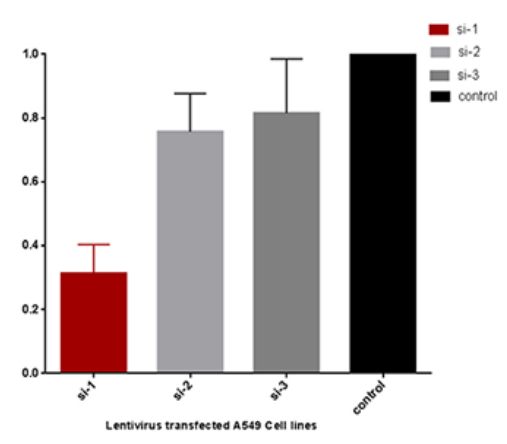

C

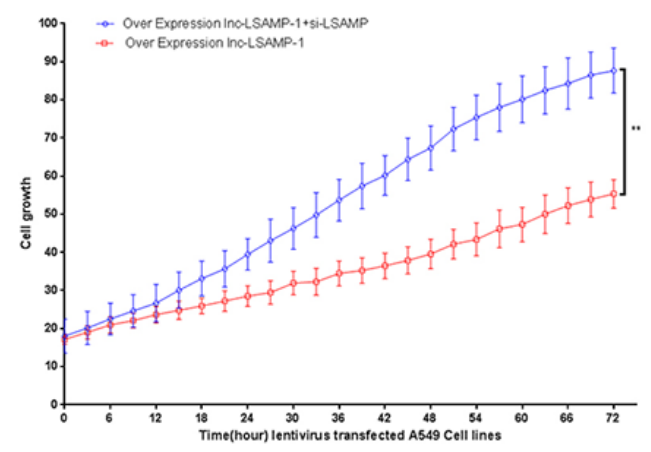

e

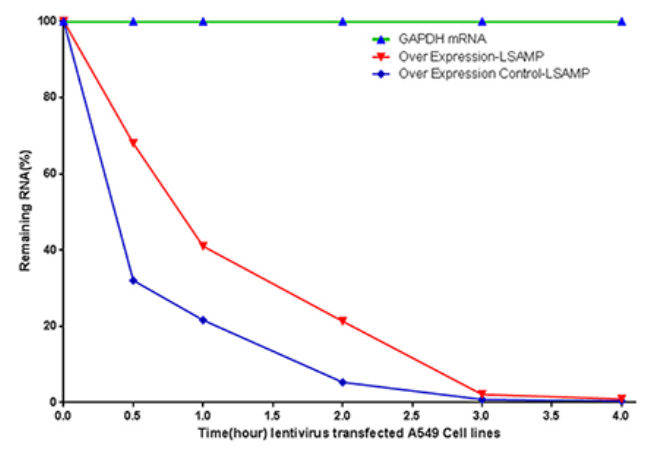

b

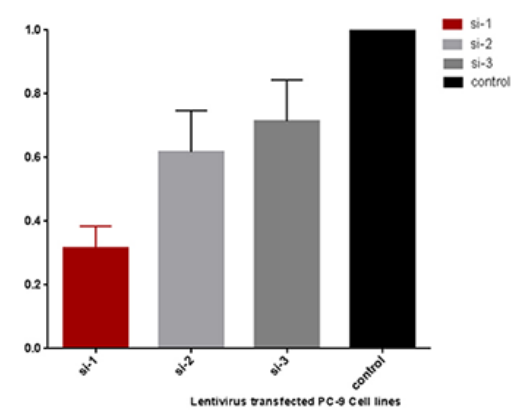

$\mathrm{d}$

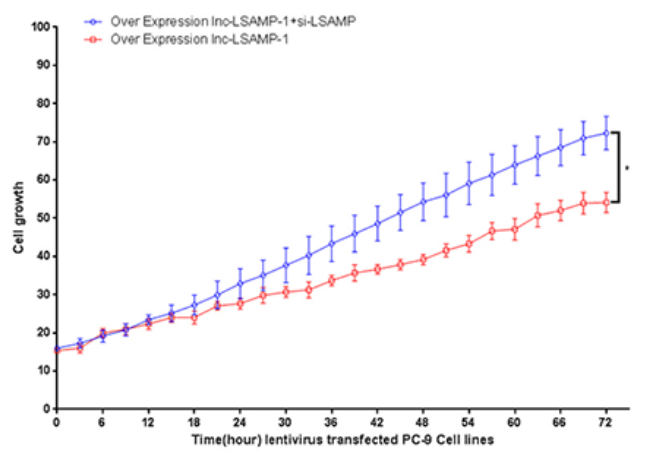

f

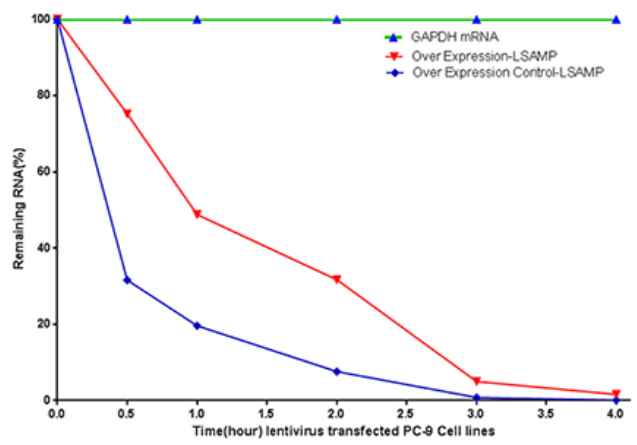

Figure 4

Figure 4

(a) (b)The efficiency of si (LSAMP gene) measured by qRT-PCR. (c) (d) The cell proliferation rate was compared with or without si-1.

(e) (f) Over-expressed A549 and PC-9 cells and control were treated with actinomycin D (the concentration of actinomycin D was 2 $\mathrm{mg} / \mathrm{L})$. 


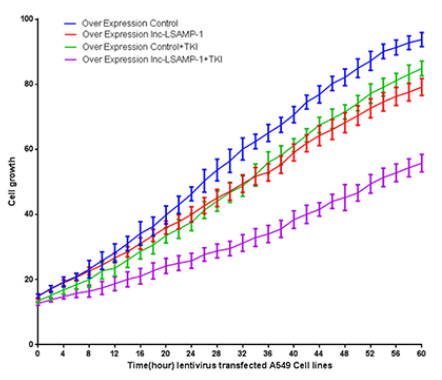

C
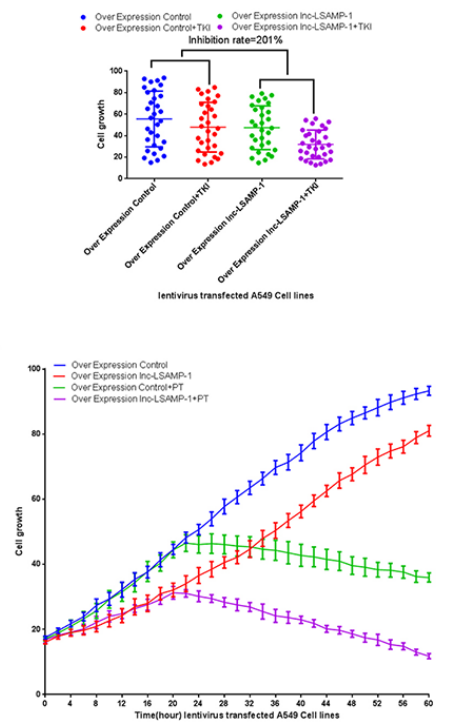

g

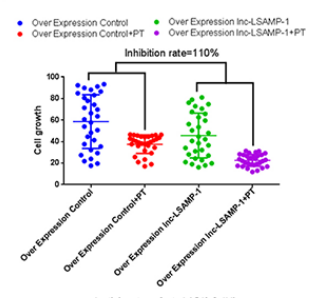

b

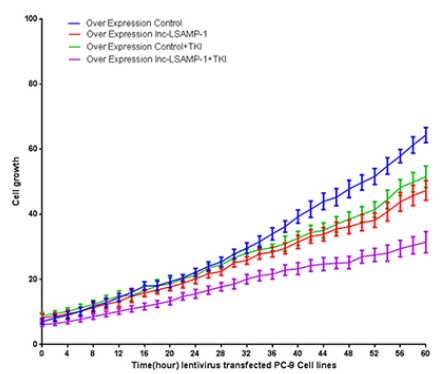

d

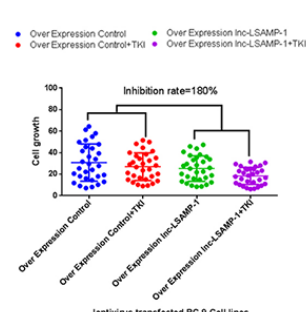

f

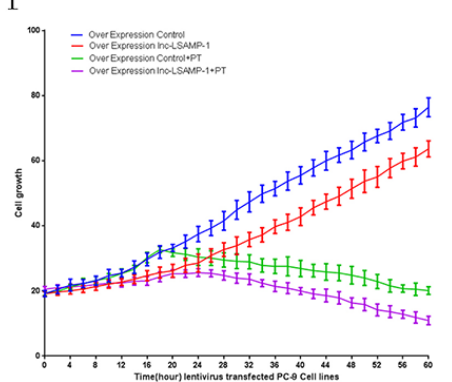

h

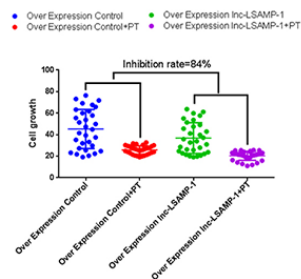

Figure 5

\section{Figure 5}

(a) (b) (c) (d) The inhibition rate of Nilotinib in A549 and PC-9 cells. (e) (f) (g) (h) The inhibition rate of carboplatin in A549 and PC-9 cells.

\section{Supplementary Files}

This is a list of supplementary files associated with this preprint. Click to download.

- FigureS2.tif

- Tables1.docx

- Figures1.tif 